HUP-96/001

DTP-96/27

hep-th/9612150

\title{
Classical integrability of the $O(N)$ nonlinear Sigma model on a half-line
}

\author{
E. Corrigan ${ }^{a}$ and Z-M Sheng ${ }^{b}$ ] \\ a. Department of Mathematical Science, University of Durham, \\ Durham, DH1 3LE, U.K. \\ b. Department of Physics, Hangzhou University, \\ Hangzhou 310028, P.R. China
}

\begin{abstract}
The classical integrability the $O(N)$ nonlinear sigma model on a half-line is examined, and the existence of an infinity of conserved charges in involution is established for the free boundary condition. For the case $N=3$ other possible boundary conditions are considered briefly.
\end{abstract}

July 1996

\footnotetext{
${ }^{1}$ email : zmsheng@whale.hzuniv.edu.cn
} 


\section{Introduction}

Recently, much attention has been paid to the study of integrable field theory with a boundary [1] [2] [5] [3] [6] [4]. The study of such field theories is not only intrinsically interesting but also provides a better understanding of boundary related phenomena in statistical physics and condensed matter [9] [10]. Probably the most famous physical example of a boundary integrable model is the Kondo problem, where the $1+1$ dimensional field theory is an effective field theory of s-wave scattering of electrons off a magnetic spin impurity. Such an impurity problem, in which one concentrates on s-wave scattering from some isolated object at the origin, generically provides interesting $1+1$ dimensional boundary field theory. There are also $1+1$ dimensional boundary quantum integrable systems of experimental relevance, such as a Luttinger liquid (Thirring) model for the edge states of electrons in the fractional quantum Hall effect 10.

An integrable field theory possesses an infinite set of independent, commuting integrals of motion. In the 'bulk theory' these integrals of motion follow from an infinite number of divergenceless currents. However, when the theory is restricted to a halfline (or to an interval) the existence of conserved charges on the whole-line does not guarantee integrability unless special boundary conditions are specified. The boundary conditions under which a theory preserves its integrability can be sought in several ways: for example, via a perturbed conformal boundary condition [11] [12] or via a Lax pair approach [4].

However, it is more difficult to discuss the integrability for non-ultralocal integrable models, such as a nonlinear sigma model on the half-line, because their fundamental Poisson bracket relations are field dependent [13 14 15 16] 174]. Ghoshal 19 has obtained the boundary S-matrix of the $\mathrm{O}(\mathrm{N})$-symmetric nonlinear sigma model, when he conjectured that this model is still integrable in the semi-infinite space with the 'free' or 'fixed' boundary conditions. However, Mourad and Sasaki 21] have concluded recently that the infinite set of non-local charges characterizing integrability on the whole line is not preserved even for the free (Neumann) boundary condition. In this paper, the question of integrability for sigma models with a boundary will be re-examined from a different view-point. First, standard tools for the model on the full line will be reviewed. In particular, the Lax pair representation, the $r, s$ matrices, and their use to generate conserved charges, will be discussed in the next section. Following the ideas of [⿶], a Lax pair on the half-line will be constructed in the third section, with the property that its zero curvature condition leads to both the equation of motion and to the boundary condition. In the fourth section, the Lax pair will be used to construct the conserved charges, suggesting integrability of the model on the half-line, and to determine compatible integrable boundary conditions. 


\section{The $O(N)$ nonlinear sigma model on the full line}

To establish notation, the standard Lax pair for the $S O(N)$ nonlinear sigma model 13. will be written in the form

$$
\begin{aligned}
& a_{0}=\frac{\lambda}{1-\lambda^{2}}\left(\lambda A_{0}+A_{1}\right) \\
& a_{1}=\frac{\lambda}{1-\lambda^{2}}\left(\lambda A_{1}+A_{0}\right)
\end{aligned}
$$

where

$$
A_{\mu}=2\left(n \otimes \partial_{\mu} n-\partial_{\mu} n \otimes n\right),
$$

and $n$ is an $N$-component, unit length vector.

It is easy to check that the zero curvature condition for the vector potentials defined by $\operatorname{eq}(1)$,

$$
\partial_{0} a_{1}-\partial_{1} a_{0}+\left[a_{1}, a_{0}\right]=0
$$

leads to the equation of motion of the model in the form

$$
n \otimes \partial^{2} n-\partial^{2} n \otimes n=0
$$

or, equivalently,

$$
\partial^{2} n_{a}+n_{a}\left(\partial_{\mu} n_{b} \partial_{\mu} n_{b}\right)=0 .
$$

The action of the $O(N)$ nonlinear sigma model may be taken to be

$$
S=\frac{1}{2} \int d^{2} x\left(\left(\partial_{\mu} n_{a}\right)^{2}+\zeta\left(n_{a}^{2}-1\right)\right)
$$

where $\zeta$ is a Lagrange multiplier. The canonical momentum associated to $n_{a}$ is $\pi_{a}=\partial_{0} n_{a}$. However, the Hamiltonian formalism is of constrained type. The Hamiltonian is

$$
H=\frac{1}{2} \int d x\left[\pi_{a}^{2}+\left(\partial_{1} n_{a}\right)^{2}\right]
$$

and the canonical Poisson brackets are

$$
\begin{aligned}
& \left\{n_{a}(x), n_{b}(y)\right\}=0 \\
& \left\{n_{a}(x), \pi_{b}(y)\right\}=\left(\delta_{a b}-n_{a} n_{b}\right) \delta(x-y) \\
& \left\{\pi_{a}(x), \pi_{b}(y)\right\}=\left(\pi_{a} n_{b}-\pi_{b} n_{a}\right) \delta(x-y)
\end{aligned}
$$

It is also useful to introduce a representation for the $S O(N)$ generators

$$
T_{\alpha \beta}^{a b}=\delta_{\alpha}^{a} \delta_{\beta}^{b}-\delta_{\beta}^{a} \delta_{\alpha}^{b},
$$


with quadratic Casimir

$$
C=\frac{1}{2} T^{a b} \otimes T^{a b}
$$

and to set, using the components (2),

$$
A_{\mu}=2 n_{a} \partial_{\mu} n_{b} T^{a b} .
$$

It is also convenient to define an additional quantity

$$
A=n_{a} T^{a b} \otimes T^{b c} n_{c} .
$$

The two operators $C$ and $A$ play a significant role in setting up the Poisson structure of the model. The use of the same notation for the matrices, or components should cause no confusion.

Using the Poisson brackets (8), and following closely Bordemann et al. [16], one may obtain the following algebraic structure:

$$
\begin{aligned}
& \left\{A_{0}(x) \stackrel{\otimes}{,} A_{0}(y)\right\}=2\left[C, I \otimes A_{0}\right] \delta(x-y) \\
& \left\{A_{1}(x) \stackrel{\otimes}{,} A_{1}(y)\right\}=0 \\
& \left\{A_{0}(x) \stackrel{\otimes}{,} A_{1}(y)\right\}=2\left[C, I \otimes A_{1}\right] \delta(x-y)-4 A(y) \delta^{\prime}(x-y) .
\end{aligned}
$$

For the last of these, use has been made of the identity

$$
(f(x)-f(y)) \delta^{\prime}(x-y)=-f^{\prime}(x) \delta(x-y) .
$$

In turn, eqs(13) may be used to calculate the Poisson brackets of the Lax pair operators themselves, casting them into the Maillet form:

$$
\begin{aligned}
&\left\{a_{1}(x, \lambda) \stackrel{\otimes}{,} a_{1}(y, \mu)\right\}=\left(\left[r(x, \lambda, \mu), a_{1}(x, \lambda) \otimes I+I \otimes a_{1}(x, \mu)\right]\right. \\
&\left.-\left[s(x, \lambda, \mu), a_{1}(x, \lambda) \otimes I-I \otimes a_{1}(x, \mu)\right]\right) \delta(x-y) \\
&+(r(x, \lambda, \mu)+s(x, \lambda, \mu)-r(y, \lambda, \mu)+s(y, \lambda, \mu)) \delta^{\prime}(x-y)
\end{aligned}
$$

where

$$
\begin{aligned}
& r(x, \lambda, \mu)=-\frac{2 \lambda \mu}{(\lambda-\mu)(1-\lambda \mu)} C+\frac{2 \lambda \mu(\lambda-\mu)(1+\lambda \mu)}{\left(1-\lambda^{2}\right)\left(1-\mu^{2}\right)(1-\lambda \mu)} A(x) \\
& s(x, \lambda, \mu)=\frac{2 \lambda \mu(\lambda+\mu)}{\left(1-\lambda^{2}\right)\left(1-\mu^{2}\right)} A(x) .
\end{aligned}
$$


In checking eqs(14) the following additional relationship is indispensable

$$
\left[A, I \otimes A_{\mu}-A_{\mu} \otimes I\right]=\left[C, A_{\mu} \otimes I\right]
$$

In equations (14), $r(x, \lambda, \mu)$ and $s(x, \lambda, \mu)$ are two matrix 'structure constants' which depend on $x$ because they depend on the field of the theory. They have following property

$$
r(x, \lambda, \mu)=-r(x,-\lambda-\mu)
$$

and satisfy the following modified Yang-Baxter equation

$$
\begin{aligned}
& {\left[(r+s)_{23}(x, \mu, \eta),(r+s)_{12}(x, \lambda, \eta)\right]+\left[(r+s)_{23}(x, \mu, \eta),(r+s)_{13}(x, \lambda, \eta)\right]} \\
& +\left[(r+s)_{13}(x, \lambda, \eta),(r-s)_{12}(x, \lambda, \mu)\right]+H_{1,23}^{(r+s)}(x, \lambda, \mu, \eta)-H_{2,13}^{(r+s)}(x, \mu, \lambda, \eta)=0,
\end{aligned}
$$

where $H_{1,23}^{r+s}(x, \lambda, \mu, \eta)$ is defined by

$$
\left\{a_{1}(x, \lambda) \otimes I,(r+s)_{23}(y, \mu, \eta)\right\}=H_{1,23}^{r+s}(x, \lambda, \mu, \eta) \delta(x-y) .
$$

One of the attractive features of the Lax pair representation is the role it plays in the generation of conserved quantities. The path-ordered exponential

$$
T(x, y ; \lambda)=\mathrm{P} \exp \int_{x}^{y} a_{1}(z, \lambda) d z
$$

enjoys the following properties,

$$
\begin{gathered}
T(x, x ; \lambda)=1, \quad T(y, x ; \lambda)=T^{-1}(x, y ; \lambda), \\
T(x, y ; \lambda) T(y, z ; \lambda)=T(x, z ; \lambda),
\end{gathered}
$$

and satisfies

$$
\begin{aligned}
& \left\{T(x, y ; \lambda) \stackrel{\otimes}{,} T\left(x^{\prime}, y^{\prime} ; \mu\right)\right\} \\
& \quad=\int_{x}^{y} d z \int_{x^{\prime}}^{y^{\prime}} d z^{\prime} T(x, z ; \lambda) \otimes T\left(x^{\prime}, z^{\prime} ; \mu\right)\left\{a_{1}(z, \lambda) \stackrel{\otimes}{,} \quad a_{1}\left(z^{\prime}, \mu\right)\right\} T(z, y, \lambda) \otimes T\left(z^{\prime}, y^{\prime} ; \mu\right) .
\end{aligned}
$$

Defining $U(\lambda)=T(-\infty,+\infty ; \lambda)$, one obtains from eq $(23)$ the relation

$$
\{U(\lambda) \stackrel{\otimes}{,} U(\mu)\}=r(-\infty, \lambda, \mu) U(\lambda) \otimes U(\mu)-U(\lambda) \otimes U(\mu) r(+\infty, \lambda, \mu) .
$$

Alternatively, assuming periodic boundary conditions on the finite interval $(-L, L)$, ie $n_{a}(-L)=n_{a}(+L)$, one has

$$
\begin{aligned}
& \left\{U_{L}(\lambda) \stackrel{\otimes}{,} U_{L}(\mu)\right\}=\left[r(-L, \lambda, \mu), U_{L}(\lambda) \otimes U_{L}(\mu)\right] \\
& \quad+\left(U_{L}(\lambda) \otimes I\right) s(L, \lambda, \mu)\left(I \otimes U_{L}(\mu)\right)-\left(I \otimes U_{L}(\mu)\right) s(L, \lambda, \mu)\left(U_{L}(\lambda) \otimes I\right),
\end{aligned}
$$


where $U_{L}(\lambda)=T(-L, L, \lambda)$. From eq $(24)$ or eq $(25)$, it is straightforward to obtain

$$
\{\operatorname{tr} U(\lambda), \operatorname{tr} U(\mu)\}=0
$$

indicating that the infinity of conserved charges obtained from $U(\lambda)$ as coefficients in an expansion in powers of $\lambda$ are in involution.

\section{Lax pair representation for $\mathrm{O}(\mathrm{N})$ sigma model on a half-line}

A Lax pair on a half-line will be constructed so that its zero curvature condition leads both to the field equation and to the boundary condition at $x=a$. The procedure developed in [4] will be followed closely. To construct a modified Lax pair including the boundary condition, it is first of all convenient to consider an additional special point $x=b(>a)$ and two overlapping regions $R_{-}: x \leq(a+b+\epsilon) / 2$; and $R_{+}: x \geq(a+b-\epsilon) / 2$. The second region will be regarded as a reflection of the first, in the sense that if $x \in R_{+}$, then

$$
n_{a}(x)=n_{a}(a+b-x) .
$$

The regions overlap in a small interval surrounding the midpoint of $[a, b]$. In the two regions, define:

$$
\begin{array}{ll}
R_{-}: & \hat{a}_{0}=a_{0}+\frac{2 \lambda}{\left(\lambda^{2}-1\right)} \theta(x-a)\left[n \otimes\left(\partial_{1} n+\mathcal{F}\right)-\left(\partial_{1} n+\mathcal{F}\right) \otimes n\right] \\
& \hat{a}_{1}=\theta(a-x) a_{1} \\
R_{+}: & \hat{a}_{0}=a_{0}+\frac{2 \lambda}{\left(\lambda^{2}-1\right)} \theta(b-x)\left[n \otimes\left(\partial_{1} n-\mathcal{F}\right)-\left(\partial_{1} n-\mathcal{F}\right) \otimes n\right] \\
& \hat{a}_{1}=\theta(x-b) a_{1}
\end{array}
$$

It is then straightforward to check this Lax pair leads to the equation of motion on the half-plane, and to the boundary conditions at the boundary point $x=a$ :

$$
\partial_{1} n_{a}=-\mathcal{F}_{a}
$$

For the moment, the question of the form of the boundary potential term which ought to be added to the Lagrangian will be postponed.

On the other hand, for $x \in R_{-}$and $x>a, \hat{a}_{1}$ vanishes and therefore the zero curvature condition merely implies that $\hat{a}_{0}$ is independent of $\mathrm{x}$, which means in turn that the fields $n_{a}$ are independent of $x$ in this region. Similar remarks apply to the region $x \in R_{+}$ and $x<b$. Hence, taking into account the reflection principle, fields are independent of $x$ throughout the interval $[a, b]$, and equal to the field value at $a$ or $b$. For general boundary conditions, the gauge potential $\hat{a}_{0}$ is different in the two regions $R_{ \pm}$. However, 
to maintain the zero curvature condition over the whole line the values of $\hat{a}_{0}$ must be related by a gauge transformation within the overlap region $[a, b]$. In principle, this gauge transformation could be quite general, even depending upon the field $n$. Here, it will be assumed a gauge transformation $\kappa(\lambda)$ exists, not depending upon either $n$, or $x^{0}$. Thus,

$$
\partial_{0} \kappa=\kappa \hat{a}_{0}\left(x^{0}, b\right)-\hat{a}_{0}\left(x^{0}, a\right) \kappa=0 .
$$

These are strong assumptions. Previous experience with affine Toda theory [4 indicates they might not lead to stronger constraints on the boundary condition than those which would be obtained by alternative means (such as examining directly the existence of conserved quantities). However, the classical $r$-matrix for affine Toda field theory does not itself depend on the Toda field. This fact represents a crucial difference. Substituting $\hat{a}_{0}$ into the above, one obtains

$$
\lambda\left[\kappa(\lambda), A_{0}\right]_{-}+[\kappa(\lambda), B]_{+}=0
$$

where

$$
B=2(n \otimes \mathcal{F}-\mathcal{F} \otimes n) .
$$

Obviously, $\kappa(\lambda)=I$ and $\mathcal{F}=0$ is one of the solutions; it corresponds to the free boundary condition. To find the others for general $N$ is less easy and only the case $N=3$ will be discussed in detail here.

It is useful first to note that when $N=3 \kappa(\lambda)$ is an element of the group $S O(3)$ and must therefore have the form

$$
\kappa_{a b}=P \delta_{a b}+Q k_{a} k_{b}+R \epsilon_{a b c} k_{c}=\kappa_{a b}^{S}+\kappa_{a b}^{A}
$$

where $P, Q, R$ and the vector $k$ could depend upon $\lambda$ but not $n$ or $x^{0}$, and the $S, A$ superscripts denote the symmetric and antisymmetric parts, respectively. Orthogonality requires

$$
P^{2}+|k|^{2} R^{2}=1 \quad|k|^{2} Q^{2}-R^{2}+2 P Q=0 .
$$

It is also useful to write

$$
\left(A_{0}\right)_{a b}=\epsilon_{a b c} \alpha_{c} \quad B=\epsilon_{a b c} \beta_{c} .
$$

Taking the symmetric and antisymmetric parts of eq(31) yields a pair of equations:

$$
\begin{gathered}
\lambda\left(\kappa_{a d}^{S} \epsilon_{d b c} \alpha_{c}+\kappa_{b d}^{S} \epsilon_{d a c} \alpha_{c}\right)+R\left(\beta_{a} k_{b}+\beta_{b} k_{a}\right)=0 \\
\lambda R\left(\alpha_{a} k_{b}-\alpha_{b} k_{a}\right)+\kappa_{a d}^{S} \epsilon_{d b c} \beta_{c}-\kappa_{b d}^{S} \epsilon_{d a c} \beta_{c}=0 .
\end{gathered}
$$

The vanishing trace of the first of eqs $(34)$ requires $R(\beta \cdot k)=0$. Therefore, assuming $R \neq 0, \beta$ must have the form

$$
\beta=\rho \times k .
$$


The second of eqs (34) forces $\rho$ to be proportional to $\alpha$ and, without any loss of generality, $\beta$ may be set equal to $\alpha \times k$. However, it is also necessary that

$$
\lambda R+2 P+|k|^{2} Q=0 .
$$

Finally, returning to the first of eqs(34) and using (35) the other components vanish provided

$$
\lambda Q+R=0 .
$$

Eqs(33), (36) and (37) (over-)determine $P, Q$ and $R$ to be

$$
P= \pm \frac{\lambda^{2}-|k|^{2}}{\lambda^{2}+|k|^{2}} \quad Q= \pm \frac{2}{\lambda^{2}+|k|^{2}} \quad R=\mp \frac{2 \lambda}{\lambda^{2}+|k|^{2}}
$$

Finally, noting

$$
\beta=2 n \times \mathcal{F} \quad \alpha=2 n \times \partial_{0} n,
$$

and, therefore,

$$
n \times \mathcal{F}=\left(n \times \partial_{0} n\right) \times k,
$$

it is straightforward to deduce

$$
\mathcal{F}=-k \times \partial_{0} n+\left(n \cdot k \times \partial_{0} n\right) n
$$

together with the further constraint:

$$
k \cdot \partial_{0} n=0 .
$$

To summarise, a consistent boundary condition requires

$$
\partial_{1} n=-k \times \partial_{0} n+\left(n \cdot k \times \partial_{0} n\right) n \quad \text { and } \quad k \cdot \partial_{0} n=0 \quad \text { at } \quad x=a .
$$

The vector $k$ may be chosen freely and represents extra parameters allowed by the boundary condition. Notice, however, that with the exception of the Neumann condition, the boundary violates the $O(3)$ symmetry of the model leaving a subgroup of rotations preserving $k$.

The choice $R=0$ leads to $\mathcal{F}=0$ and $\kappa=I$.

\section{Construction of conserved quantities}

The patching matrix $\kappa(\lambda)$ is an essential ingredient in the construction of conserved quantities for the model defined on the half-line $x \leq a$ via the trace of the generating function:

$$
U(\lambda)=T(-\infty, a ; \lambda) \kappa(\lambda) T(b, \infty ; \lambda) .
$$


Using the reflection properties,

$$
\begin{array}{cc}
n(x)=n(a+b-x) & \partial_{1} n(x)=-\partial_{1} n(a+b-x) \\
\partial_{0} n(x)=\partial_{0} n(a+b-x) & a_{1}(x, \lambda)=-a_{1}(a+b-x,-\lambda),
\end{array}
$$

eq(44) may be rewritten as

$$
U(\lambda)=T(-\infty, a ; \lambda) \kappa(\lambda) T^{-1}(-\infty, a ;-\lambda) .
$$

Integrability requires the conserved quantities to be in involution and, when the model is restricted to a half-line, this in turn implies a compatibility relation which must be satisfied. This relation is expected to involve $\kappa(\lambda)$ and the classical $r$ matrix entering eq(14). The Poisson brackets:

$$
\begin{gathered}
\left\{T(x, y ; \lambda) \stackrel{\otimes}{,} T^{-1}(x, y ; \mu)\right\}=(T(x, y ; \lambda) \otimes I) r(y, \lambda, \mu)\left(I \otimes T^{-1}(x, y ; \mu)\right) \\
-\left(I \otimes T^{-1}(x, y ; \mu)\right) r(x, \lambda, \mu)(T(x, y ; \lambda) \otimes I), \\
\left\{T^{-1}(x, y ; \lambda) \stackrel{\otimes}{,} T(x, y ; \mu)\right\}=-\left(T^{-1}(x, y ; \lambda) \otimes I\right) r(x, \lambda, \mu)(I \otimes T(x, y ; \lambda)) \\
+(I \otimes T(x, y ; \lambda)) r(y, \lambda, \mu)\left(T^{-1}(x, y ; \mu) \otimes I\right),
\end{gathered}
$$

and

$$
\begin{gathered}
\left\{T^{-1}(x, y ; \lambda) \stackrel{\otimes}{,} T^{-1}(x, y ; \mu)\right\}=\left(T^{-1}(x, y ; \lambda) \otimes T^{-1}(x, y ; \mu)\right) r(x, \lambda, \mu) \\
-r(y, \lambda, \mu)\left(T^{-1}(x, y ; \lambda) \otimes T^{-1}(x, y ; \mu)\right)
\end{gathered}
$$

imply the following Poisson bracket for a pair of generating functions, as defined in eq(45) but with different parameters $\lambda$ and $\mu$,

$$
\begin{gathered}
\{U(\lambda) \stackrel{\otimes}{,} U(\mu)\}=r(-\infty, \lambda, \mu)(U(\lambda) \otimes U(\mu))-(U(\lambda) \otimes U(\mu)) r(-\infty, \lambda, \mu) \\
+(U(\lambda) \otimes I) r(-\infty, \lambda,-\mu)(I \otimes U(\mu))-(I \otimes U(\mu)) r(-\infty, \lambda,-\mu)(U(\lambda) \otimes I) \\
+(T(\lambda) \otimes T(\mu))[-r(a, \lambda, \mu) \kappa(\lambda) \otimes \kappa(\mu)+\kappa(\lambda) \otimes \kappa(\mu) r(a, \lambda, \mu) \\
+(I \otimes \kappa(\mu)) r(a, \lambda,-\mu)(\kappa(\lambda) \otimes I)-(\kappa(\lambda) \otimes I) r(a, \lambda,-\mu)(I \otimes \kappa(\mu))] . \\
\cdot\left(T^{-1}(-\lambda) \otimes T^{-1}(-\mu)\right)
\end{gathered}
$$

where $T(\lambda)=T(-\infty, a ; \lambda)$. To establish

$$
\{\operatorname{tr} U(\lambda), \operatorname{tr} U(\mu)\}=0
$$


it would be sufficient to require

$$
\begin{aligned}
& {[\kappa(\lambda) \otimes \kappa(\mu), r(a, \lambda, \mu)]+(I \otimes \kappa(\mu)) r(a, \lambda,-\mu)(\kappa(\lambda) \otimes I)} \\
& -(\kappa(\lambda) \otimes I) r(a, \lambda,-\mu)(I \otimes \kappa(\mu))=0
\end{aligned}
$$

a condition reminiscent of those encountered in [1] and [4]. Obviously, eq(51) is satisfied by $\kappa(\lambda)=I$, corresponding to the free boundary condition. However, it is not in general satisfied for the other possibilities, principally because $\kappa$ is independent of $n$ while $r$ depends explicitly on the field $n$ but not its derivatives.

Alternatively, to demonstrate eq(50) it would be sufficient to require

$$
\left[\kappa(\lambda), T^{-1}(-\lambda) T(\lambda)\right]=0 .
$$

However, while no proof (or disproof) of this relation is available, it too would appear to be unlikely to be true given the conjectured $n$-independence of $\kappa$.

\section{Conclusion and Discussion}

It has been argued that at the classical level the $O(N)$ nonlinear sigma models remain integrable when restricted to a half-line with a Neumann boundary condition. Infinitely many conserved charges in involution have been constructed via eq(41) and one might suppose this result to be a strong indicator of integrability. However, Mourad and Sasaki 21] have taken a different view, analysing the non-local charges for the theory restricted to a half-line, and concluding the opposite. Their discovery of the absence of non-local charges in the presence of the Neumann condition would appear to supply strong reasons for a lack of integrability for even the simplest of boundary conditions. On the other hand, others (for example, Ghoshal [19] and Mackay [20]) have assumed the sigma models restricted to a half-line are quantum integrable, and have conjectured quantum reflection matrices compatible with the reflection bootstrap. One would expect the quantum integrability to be reflected in classical features of these theories (although it must be borne in mind that the quantum sigma models have a spectrum and other properties which are very far from being reflected in the classical degrees of freedom). It would seem one might be forced to conclude that the classical non-local charges are not crucial for integrability.

It has been shown that the $O(3)$ sigma model on a half-line has a consistent Lax pair description not only for the Neumann condition at the boundary, but also for some other suitably chosen conditions, reported in (43). However, it is not yet clear if these alternative boundary conditions allow charges in involution. These conditions also present 
something of a puzzle because they do not appear to be derivable from a boundary potential added to the bulk Lagrangian. The condition (41) would be fine by itself and follow from the boundary potential

$$
\mathcal{B}=\frac{1}{2} n \cdot k \times \partial_{0} n+\frac{\tau}{2}\left(n^{2}-1\right),
$$

where $\tau$ is a Lagrange multiplier. However, the additional constraint (42) needs to be imposed 'by hand'. The significance of this is not really clear. However, this fact and the remarks above suggest that the Neuamnn condition is the only boundary condition compatible with the classical Poisson structure.

\section{Acknowledgements}

One of us (ZMS) would like to thank the Department of Mathematical Sciences, University of Durham for kind hospitality, the Pao Foundation for a Fellowship, and the National Natural Science Foundation of China for support. The other (EC) would like to thank Peter Bowcock, Patrick Dorey and Alistair MacIntyre for comments.

\section{References}

[1] E.K. Sklyanin, Boundary conditions for integrable quantum systems, J. Phys. A21 (1988) 2375-2389.

[2] S. Ghoshal and A.Zamolodchikov, Boundary $S$ matrix and boundary state in twodimensional integrable quantum field theory Int. J. Mod. Phys. A9 (1994) 3841.

[3] E. Corrigan, P.E. Dorey, R.H. Rietdijk and R. Sasaki, Affine Toda field theory on a half-line, Phys. Lett. B333 (1994)83.

[4] P. Bowcock, E. Corrigan, P.E. Dorey and R.H. Rietdijk, Classically integrable boundary conditions for affine Toda field theory, Nucl. Phys. B 445(1995)469.

[5] S. Ghoshal, Boundary state boundary $S$ matrix of the sine-Gordon model Int. J. Mod. Phys. A9 (1994) 4801.

[6] S. Skorik and H. Saleur, Boundary bound states and boundary bootstrap in the sine-Gordon model with Dirichlet boundary condition, J. Phys. A28 (1995) 6605.

[7] S. Penati, D. Zanon, Quantum Integrability in two -dimensional system with boundary, Phys. Lett. B358 (1995)63. 
[8] Z.M. Sheng and H.B. Gao, On the sine-Gordon-Thirring equivalence in the presence of a boundary, DTP-95/43, hep-th/9512011, Int. J. Mod. Phys. A11 (1996)4089.

[9] A. LeClair, Quantum Theory of Self-Induced Transparency, Nucl. Phys. B450 (1995) 753.

[10] X.G. Wen, Edge transport properties of the fractional quantum Hall states and weak-impurity scattering of a one-dimensional density wave Phys. Rev. B44 (1991) 5708 .

[11] J.L. Cardy, Boundary condition, fusion rules and the Verlinde formula, Nucl. Phys. B324 (1989) 581.

[12] J.L. Cardy and D.C. Lewellen, Bulk and boundary operators in conformal field theory, Phys. Lett. B259 (1991) 274.

[13] K. Pohlmeyer, Integrable Hamiltonian systems and interactions through constraints, Commun. Math. Phys. 46 (1976) 207.

[14] M. Lüscher and K. Pohlmeyer, Scattering of massless lumps and non-local charges in the two-dimensional classical nonlinear sigma model, Nucl. Phys. B137 (1978) 46.

[15] J.M. Maillet, Kac-Moody algebra and extended Yang-Baxter relations in the nonlinear sigma model, Phys. Lett. B162 (1985) 137; New integrable canonical structures in two-dimensional models, Nucl. Phys B69 (1986) 54-76.

[16] M. Bordemann, M. Forger, J. Laartz and U. Schäper, The Lie-Poisson structure of integrable classical nonlinear sigma models, Commun. Math. Phys. 152 (1993)167190.

[17] B.Y. Hou, B.Y. Hou, Y.W. Li and B. Wu, The Poisson-Lie structure of nonlinear $O(N)$ sigma model by using the moving frame method, J. Phys. A27 (1994) 72097216

[18] A.O. Garcia, R.C. Trinchero, Constructive building of the Lax pair in the non-linear sigma model, hep-th/951102.

[19] S. Ghoshal, Boundary S-matrix of the $O(N)$ symmetric nonlinear sigma model, Phys, Lett. B334 (1994) 363.

[20] N.J. Mackay, Fusion of $S O(N)$ reflection matrices, Phys. Letts. B357 (1995) 89.

[21] M.F. Mourad and R. Sasaki, Non-linear Sigma models on a half plane, YITP-95-4, hep-th/9509153. 